\title{
BRANCHING STRUCTURE OF MOTONEURON STEM DENDRITES: A STUDY OF NECK MUSCLE MOTONEURONS INTRACELLULARLY STAINED WITH HORSERADISH PEROXIDASE IN THE CAT ${ }^{1}$
}

\author{
P. K. ROSE ${ }^{2}$
}

\author{
Department of Physiology, Queen's University, Kingston, Ontario, Canada K7L 3N6
}

Received March 16, 1982; Revised May 20, 1982; Accepted May 21, 1982

\begin{abstract}
The branching structure of the stem dendrites of five motoneurons innervating the dorsal neck muscles, biventer cervicis and complexus, was examined in the adult cat using intracellular staining techniques. The dendritic tree of each motoneuron was reconstructed completely and then dissected into several parts, each corresponding to the branches belonging to a single stem dendrite. Each stem dendrite then was rotated into at least one other plane to determine the three-dimensional distribution pattern of its branches. Twenty-five of the 49 stem dendrites examined had branches. which were confined to a small region of the territory occupied by the complete dendritic tree. These dendrites were distributed to one of three zones, a ventral zone deep in the ventral horn, a lateral zone dorsolateral to the motor nucleus, and a medial zone dorsomedial to the motor nucleus. The remaining stem dendrites projected to two or more of these zones and occasionally occupied a region almost as large as the territory occupied by the complete dendritic tree. The frequency of each type of stem dendrite, defined according to the number and location of the zones to which they projected, was remarkably consistent from motoneuron to motoneuron.

The maximum order of branching reached by stem dendrites of biventer cervicis and complexus motoneurons was variable. Stem dendrites whose maximum order of branching was four or less usually had one major stalk from which emerged simple, unbranched, side processes. The branching pattern of stem dendrites whose maximum order of branching was five or greater was more complex. Nevertheless, the pattern of branching of the distal regions of these stem dendrites was similar to that of stem dendrites whose maximum order of branching was four or less. The branching structure was not related, in a simple manner, to the dendritic distribution pattern.

These results indicate that the distribution and branching structure of motoneuron stem dendrites are organized in a complex, but precise, fashion which could play an important role in the integrative properties of the motoneuron.
\end{abstract}

Much of our understanding of motoneuron dendritic function is based on mathematical models which examine the effect of synaptic inputs arranged at discrete locations on a single unbranched cable (Jack et al., 1975; Rall, 1977). Although these models have proven very useful in predicting the relative influence of synapses located on proximal versus distal dendrites, comparatively little is known about the consequences of variations in dendritic

\footnotetext{
${ }^{1}$ The research was supported by the Canadian Medical Research Council and the Botterell Foundation at Queen's University. I wish to thank Ms. M. Neuber for excellent technical assistance and Drs. F. J. Richmond, P. Zarzecki, and V. C. Abrahams for many helpful comments.

${ }^{2}$ To whom correspondence should be addressed at Department of Physiology, Botterell Hall, Queen's University, Kingston, Ontario, Canada K7L 3N6.
}

branching structure and the effects of synaptic inputs differentially distributed within a complex dendritic tree. Using a model with incorporated branching dendrites, Rall (1964) found that several inputs arranged on different dendritic branches were more effective than the same inputs localized to one branch (see also Rinzel and Rall, 1973). More recently, Rall and Rinzel (1973) reported that increasing the maximum order of branching increases the attenuation of the synaptic potentials of distal synapses even though the dendritic electrotonic length is not changed. These examples illustrate that the complex branching structure of motoneuron dendrites may be an important factor in determining the integrative properties of the motoneuron. However, without experimental morphological data on the branching structure of motoneuron dendrites and the distribution of synaptic inputs, the predictions derived from models can demonstrate 
only the potential role of dendritic branching in motoneuron operation.

This study is concerned with the distribution and branching structure of motoneuron dendrites. Most studies of motoneuron structure have used staining techniques which limited the description of the dendritic tree to the dendritic branches caputured in one or two histological sections (Ramon y Cajal, 1909; Sprague and Ha, 1964; Romanes, 1964; Scheibel and Scheibel, 1966, 1967, 1969, 1970a; Szekely, 1976; Light and Metz, 1978). More recent studies of motoneuron morphology have used intracellular staining techniques which permitted the reconstruction of all dendrites belonging to a single neuron (Lux et al., 1970; Lux and Schubert, 1975; Barrett and Crill, 1974; Burke et al., 1979; Egger et al., 1980; Rose, 1981; Rose and Richmond, 1981; Brown and Fyffe, 1981; Ulfhake and Kellerth, 1981; Ulfhake and Cullheim, 1981). Nevertheless, even with these techniques, it is difficult to follow the course and branching structure of individual dendrites, since the remarkable complexity of the complete dendritic tree obscures individual dendrites.

To solve these problems, dendritic trees of motoneurons injected with horseradish peroxidase (HRP) were subdivided into several parts. Each part corresponded to a stem dendrite which included all of the branches originating from a single primary dendrite. This simple procedure provided a much clearer picture of the threedimensional distribution and branching structure of motoneuron dendrites. This paper describes the morphology of the stem dendrites of motoneurons innervating the dorsal neck muscles, biventer cervicis and complexus. The distribution pattern of their complete dendritic trees has been described previously (Rose, 1981). Some of the results have been presented in abstract form (Rose and Twiddy, 1981).

\section{Materials and Methods}

Experiments were performed on five adult cats weighing between 2.7 and $3.3 \mathrm{~kg}$. All animals were anesthetized with $\alpha$-chloralose ( $70 \mathrm{mg} / \mathrm{kg}$, i.v.) following the induction of anesthesia with ethyl chloride and ether. After the spinal cord was exposed from $\mathrm{C} 1$ to $\mathrm{C} 4$, the cat was paralyzed with gallamine triethiodide (Flaxedil, Poulenc; 2.5 to $5.0 \mathrm{mg} / \mathrm{kg} / \mathrm{hr}$ ) and artificially ventilated. A bilateral pneumothorax was performed routinely to reduce respiratory related movements. End tidal $\mathrm{PCO}_{2}$ was monitored and maintained at 3.5 to $4.0 \%$ by adjusting either the tidal volume or respiratory rate. Rectal temperature was maintained at $37 \pm 1^{\circ} \mathrm{C}$ using a feedback-controlled heating pad.

The methods of intracellular recordings, staining histological procedures, and dendritic tree reconstructions were identical to those used previously (Rose, 1981; Rose and Richmond, 1981).

The origin of a stem dendrite usually was easily identified by a conical protrusion from the soma which tapered to a cylindrical shaft (Fig. 1). However, the exact origin of a small number of stem dendrites was equivocal due to either the plane of section (for example, dendrites 3 and 8, Fig. 1), overlapping dendrites (for example, dendrites 6,9 , and 11, Fig. 1), or proximal bifurcations (for example, dendrite 7, Fig. 1). As a consequence, in
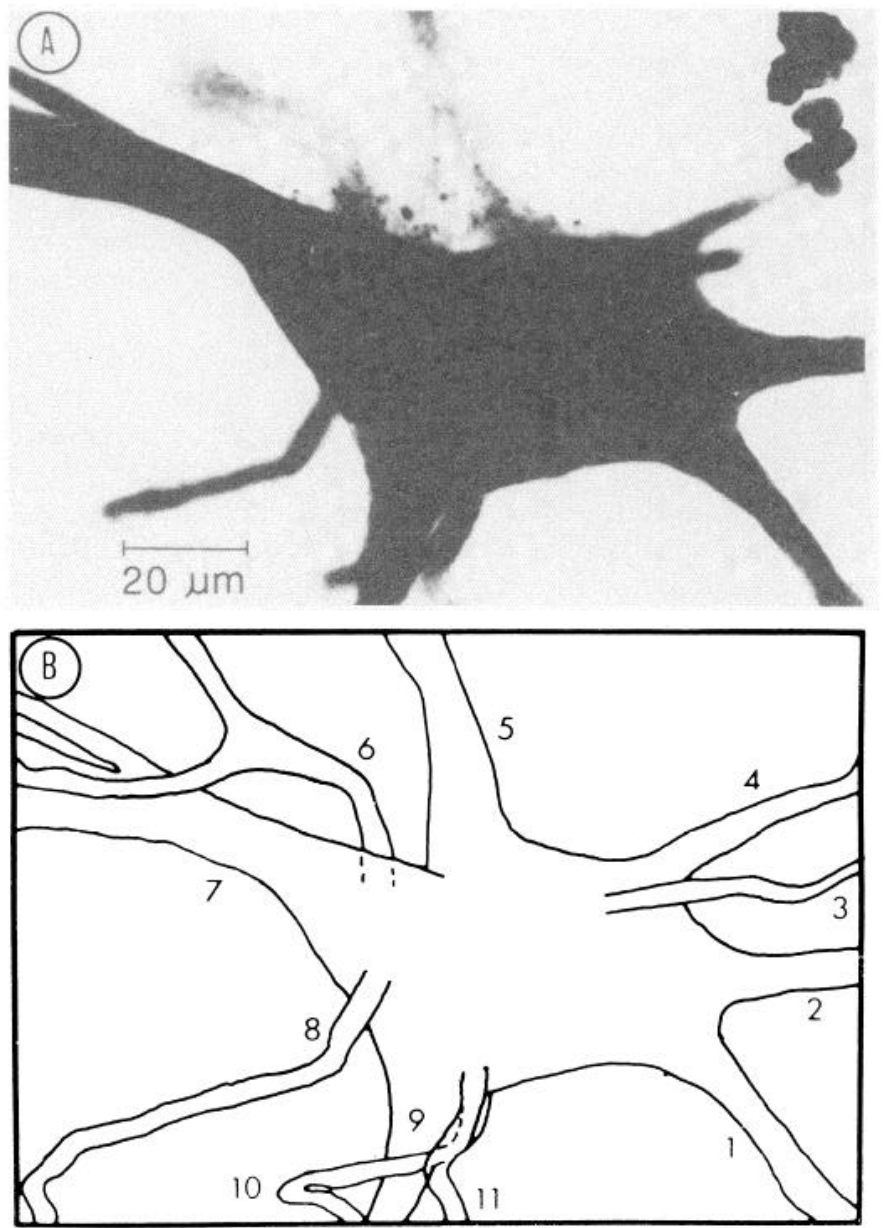

Figure 1. A, Photomicrograph of the cell body of a biventer cervicis and complexus motoneuron. Note the conically shaped origin of many of the dendrites. $B$, Camera lucida drawing of the soma and proximal dendrites of the neuron shown in $A$. This drawing was obtained by superimposing the soma and dendrites shown in the photomicrograph above on the dendritic profiles contained in the adjacent histological section. Note that the exact origins of dendrites 6,10 , and 11 are difficult to determine from the photomicrograph.

this study, each dendrite which originated in the vicinity of the soma and formed a smooth, cylindrical shaft was considered to be the primary branch of a stem dendrite.

Beginning at the cell body, each stem dendrite was traced from composite drawings of the complete dendritic tree. Overlapping dendrites were color-coded to ensure that they would not be confused with dendritic bifurcations. In addition, all dendritic bifurcations and terminals were numbered according to the histological section on which they were found. These numbers were used to reconstruct a view of each stem dendrite in at least one additional plane. This procedure was extremely useful when comparing the three-dimensional distribution of stem dendrites from different neurons which had been sectioned histologically in different planes.

\section{Results}

This report describes the characteristics of 49 stem dendrites which originated from five biventer cervicis and complexus motoneurons. These motoneurons were 
selected because the dark brown or black HRP reaction product in distal dendrites ended abruptly at what was presumably the dendritic terminal.

Distribution of stem dendrites. Stem dendrites of biventer cervicis and complexus motoneurons were distributed in a variety of patterns. The branches of some stem dendrites only projected into a small region of the territory occupied by the complete dendritic tree. Other stem dendrites, however, had much larger distribution patterns. In order to compare the distribution patterns of stem dendrites belonging to the same neuron, as well as those belonging to other motoneurons, the ventral horn was divided into four zones (Fig. 2). The locations of three of these zones were chosen to correspond to the territories occupied by the three major dendritic projections (ventral, dorsolateral, and dorsomedial) of biventer cervicis and complexus motoneurons (Rose, 1981). The fourth zone was located betwee the "V" formed by the other three zones and contained few dendrites from biventer cervicis and complexus motoneurons.

Figures 3 to 5 show examples of stem dendrites whose branches all lay within the ventral zone. These stem dendrites formed a major component of the rostrally and caudally directed dendrites characteristic of the whole dendritic tree. Their proximal and intermediate branches usually followed relatively straight paths. In contrast, their distal branches frequently followed a more tortuous path and occasionally terminated near the most dorsal border of the ventral zone (Fig. 3, terminal a). Some stem dendrites bifurcated close to the soma and sent branches both rostrally and caudally. These stem dendrites also had distal branches which terminated close to the soma, usually in the ventrolateral or ventromedial funiculus (Fig. 4, terminal c). These branches originated from proximal dendrites and followed a tortuous path in the vicinity of the soma. A few stem dendrites whose branches were restricted to the ventral zone did not have

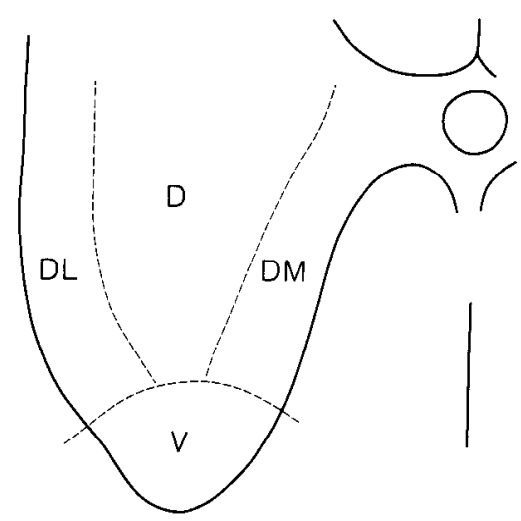

Figure 2. Low power drawing of the C3 ventral horn. The dashed lines subdivide the ventral horn into four zones. These zones correspond to the regions used to describe the distribution of the stem dendrites from biventer cervicis and complexus motoneurons. The ventral $(V)$ zone contained dendrites traveling rostrally and caudally and corresponded to the ventral part of lamina VII. Dorsomedial and dorsolateral dendrites were found in the zones designated $D M$ and $D L$, respectively. These zones included the adjacent white matter as well as part of laminae VI, VII, and VIII. The dorsal $(D)$ zone corresponded to central regions of laminae VII and VIII. long rostral or caudal projections (Fig. 5). These dendrites only projected into the ventral or ventrolateral funiculus and resembled the local tortuous branches of the more complex stem dendrites which also had long rostrally and caudally directed dendrites.

Each motoneuron had at least one stem dendrite whose branches were restricted to the dorsolateral zone. Two distribution patterns were found. In one pattern, the dendrites were arranged in a narrow rostral-caudal region directly lateral and dorsolateral to the soma (Fig. 6). These branches originated from a primary dendrite which projected directly lateral or dorsolateral toward the spinal accessory nucleus. Most of the subsequent dendritic branching occurred in or dorsal to the spinal accessory nucleus. Secondary and tertiary branches either followed a lateral path into the lateral funiculus or a dorsal path into laminae VII and VIII. The second distribution pattern was more complex and had a much wider rostral and caudal distribution (Fig. 7). The primary dendrite divided in the ventral zone close to the soma and at least one of the resulting branches traveled rostrally or caudally for 300 to $600 \mu \mathrm{m}$ before reaching the spinal accessory nucleus. Several more dendritic branches frequently emerged along this route. These branches usually traveled parallel to the first branch before arching laterally and dorsally into the spinal accessory nucleus. As a consequence, these stem dendrites, when reconstructed in the horizontal plane, resembled an open fan or chalice whose base was located at the soma. After reaching the spinal accessory nucleus, most dendrites turned dorsally to terminate in laminae VII and VIII.

Stem dendrites confined to the dorsomedial zone all followed the same distribution pattern (Fig. 8). Dendritic branches were directed either toward the central canal or medially into the ventromedial funiculus. Occasionally, dendrites in the ventromedial funiculus turned dorsally and laterally to loop back into the medial part of lamina VIII. Few dendrites traveled rostrally or caudally and, as a consequence, the rostrocaudal extent of these stem dendrites was much smaller than their counterparts in the lateral part of the ventral horn.

All of the remaining stem dendrites had a wider distribution pattern and occupied two or three zones. Stem dendrites whose branches were either distributed in two zones usually had a ventral zone projection and either a dorsomedial or dorsolateral zone projection. In most respects, the distribution pattern of these stem dendrites was a simple, spatial combination of stem dendrites whose branches were restricted to a single zone and which have been described previously. For example, many of these stem dendrites had major rostral or caudal projections which branched extensively in the ventral zone in a manner similar to that of stem dendrites whose branches were restricted to the ventral zone. However, dendrites in the dorsomedial and dorsolateral zones often followed a longer rostral or caudal path than usually was observed for stem dendrites whose branches were restricted to the dorsolateral and dorsomedial zones. As a consequence, the dorsolateral and dorsomedial projections often occupied regions rostral and caudal to the soma which were not reached by stem dendrites whose 


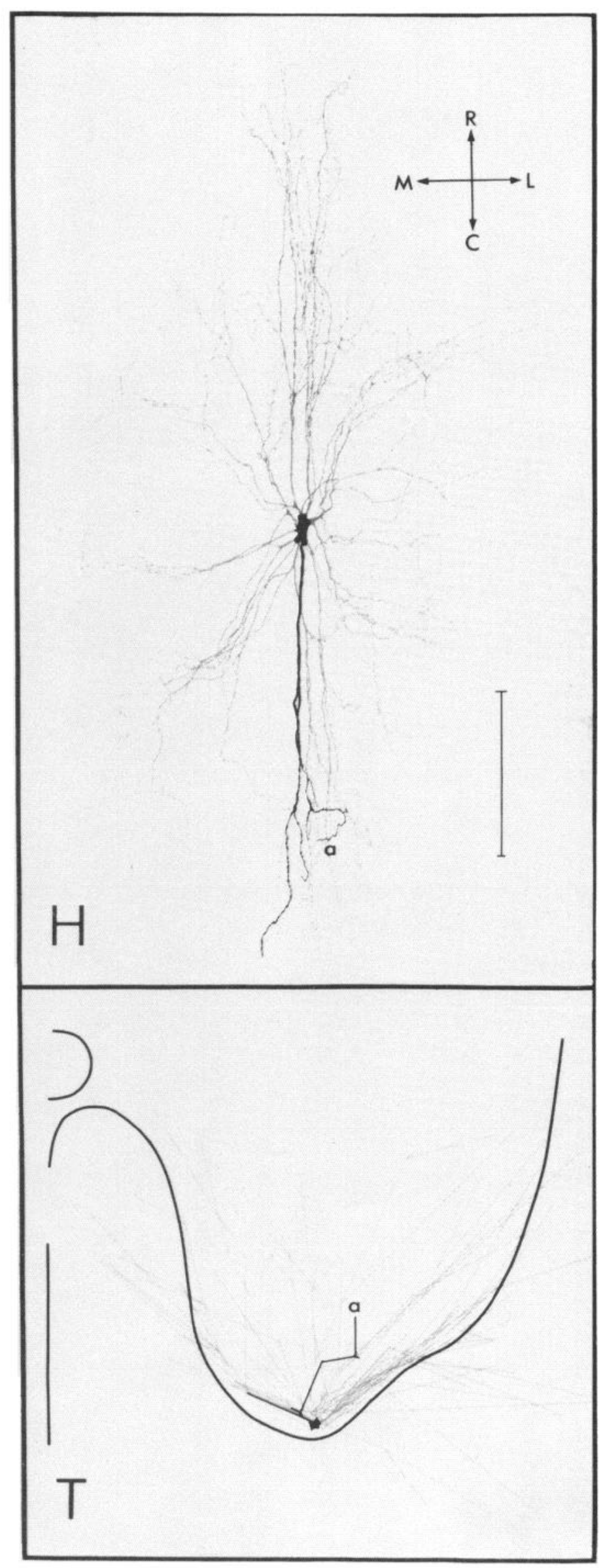

branches were restricted to the dorsolateral or dorsomedial zones.

Each cell examined had at least 1 stem dendrite which projected to three zones. The most common combination consisted of dendrites in the ventral, dorsolateral, and dorsomedial zones (Fig. 9). Like stem dendrites located in two zones, the distribution of dendritic branches in each zone was similar to the distribution of stem dendrites whose branches were restricted to that zone. Most projections in the ventral zone consisted of numerous branches which projected either rostrally or caudally. The proximal paths of dendrites projecting to the dorsolateral and dorsomedial zones usually followed a long rostral or caudal route in the ventral zone, similar to the projection pattern of two-zone stem dendrites projecting to the ventral zone and either the dorsomedial or dorsolateral zones.

The frequency of each stem dendrite distribution pattern was remarkably consistent from motoneuron to motoneuron (Table I). Each motoneuron usually had at least 1 stem dendrite whose branches were restricted to the ventral zone, dorsolateral zone, or dorsomedial zone. Stem dendrites projecting to two zones most frequently involved combinations of the ventral and dorsomedial zones or the ventral and dorsolateral zones. All but one motoneuron had 1 or more stem dendrite whose branches were distributed in the ventral, dorsolateral, and dorsomedial zones. Few dendrites projected into the dorsal zone either alone or in combination with other zones. The absence of any stem dendrites projecting to the dorsolateral zone in combination with only the dorsomedial zone was unexpected, since both of these zones contain a large number of biventer cervicis and complexus motoneuron dendrites.

Branching structure. The highest order of dendritic branching reached by each stem dendrite is shown in Figure 10. Some stem dendrites did not branch at all or only branched one or two times. Other stem dendrites had up to seven or eight orders of branching. This variability was not related to the zone in which the stem dendrite was located and was only partly related to the number of zones in which a stem dendrite projected. Most stem dendrites whose maximum order of branching was less than six were single zone stem dendrites. However, many other single zone stem dendrites had seventh or eighth order dendritic branches similar to stem dendrites which projected to two or three zones.

Figure 3. Distribution of a stem dendrite whose branches were restricted to the ventral zone. In this figure and in Figures 4 to 9 , the branches of each stem dendrite are shown in two orthogonal planes to illustrate their three-dimensional distribution. One of the drawings shows the stem dendrite in the plane in which the neuron was reconstructed and the other drawing shows a stick figure representation of the stem dendrite rotated into an orthogonal plane. Each drawing shows the stem dendrite superimposed on a phantom of the whole dendritic tree. This procedure provided a set of landmarks to illustrate the contribution of each stem dendrite to the whole dendritic tree. The lowercase letters in each figure indicate corresponding dendritic terminals. All drawings are at the same magnification. Calibration bar, $500 \mu \mathrm{m}$. The following abbreviations apply to Figures 3 to 9 : $C$, caudal; $D$, dorsal; $H$, horizontal; $L$, lateral; $M$, medial; $R$, rostral; $S$, sagittal; $T$, transverse; $V$, ventral. 


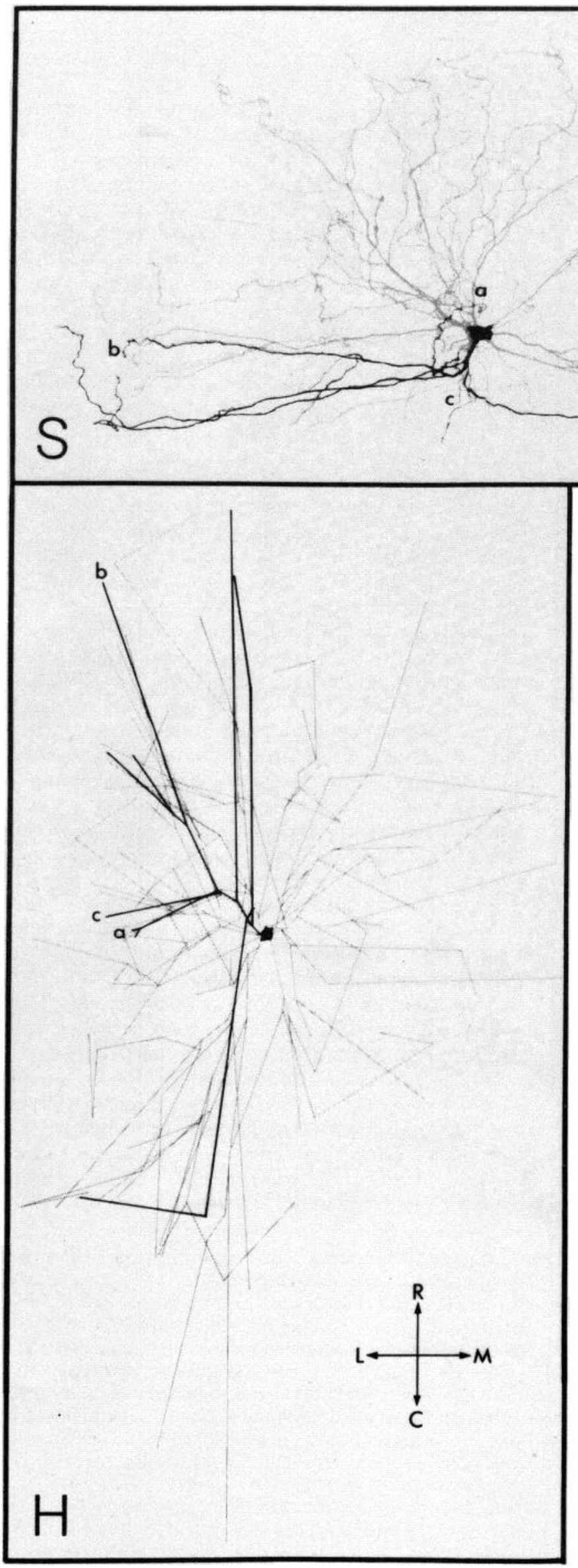

Figure 4. Distribution of a stem dendrite whose branches were restricted to the ventral zone and projected both rostrally and caudally. 


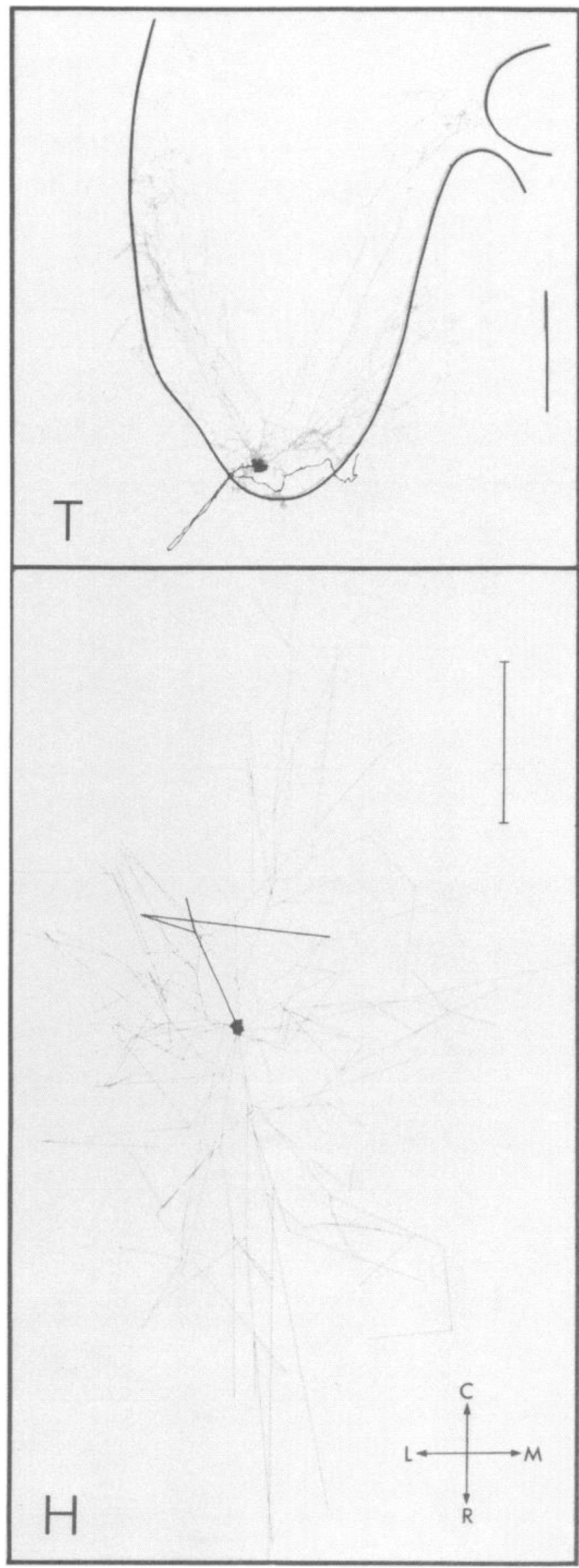

The branching structure of stem dendrites of biventer cervicis and complexus motoneurons did not follow a single pattern but depended on the maximum order of branching reached by the stem dendrites. Stem dendrites whose maximum order of branching was four or less usually formed a simple tree which consisted of a central stalk and unbranched side processes (Fig. 11). Stem dendrites whose maximum order of branching was greater than four had a more complex branching structure (Fig. 11). Most lower order branches did branch, but these branches then followed the branching pattern of stem dendrites with a maximum order of branching of four or less. As a consequence, many of these dendrites appeared to be composed of two or more stalks, each with side processes which either did not branch or only branched once. One of these stalks almost invariably reached a higher order of branching than any of the others.

The division of the dendritic branching structure into several separate stalks was not related, at least in a simple manner, to the dendritic distribution pattern of stem dendrites which projected into two or more zones. All of the branches of some stalks were confined to a single zone (Fig. 12, $A$ and $C$ ). Occasionally, if the branching structure was organized into two stalks, each stalk occupied a different territorial domain (Fig. 12A). However, many stalks had branches which projected to two or more zones. As a consequence, branches from different stalks shared the same zone (Fig. 12, $B$ and $C$ ). This distribution was the result of either last order branches projecting to different zones or higher order dendrites projecting to one zone but lower order dendrites, belonging to the same stalk, projecting to a different zone.

\section{Discussion}

Motoneurons innervating the dorsal neck muscles, biventer cervicis and complexus, have remarkably similar dendritic trees which differ from those of neighboring motoneurons (Keirstead and Rose, 1980; Rose, 1981; Rose et al., 1981). The results of the present study illustrate that the precise arrangement of biventer cervicis and complexus dendrites is not confined to the whole dendritic tree but also extends to the organization of the stem dendrites. Most stem dendrites were arranged in one of six distribution patterns and the frequency of each type of distribution pattern was relatively constant from motoneuron to motoneuron. The branching structure also was organized in a precise fashion. Almost all stem dendrites had a markedly asymmetrical branching structure which was composed of one or more stalks which gave rise to simple side branches.

In the present experiments, the dendritic distribution pattern of biventer cervicis and complexus motoneurons was used to divide the ventral horn into four zones, three of which corresponded to their three major dendritic projections. Some of these zones also correspond to the terminal regions of primary afferents and descending systems which make monosynaptic connections on these motoneurons. For example, the lateral vestibular spinal tract terminates in the medial part of the ventral horn in

Figure 5. Distribution of a stem dendrite whose branches arborized in the vicinity of the cell body in the ventral zone. 


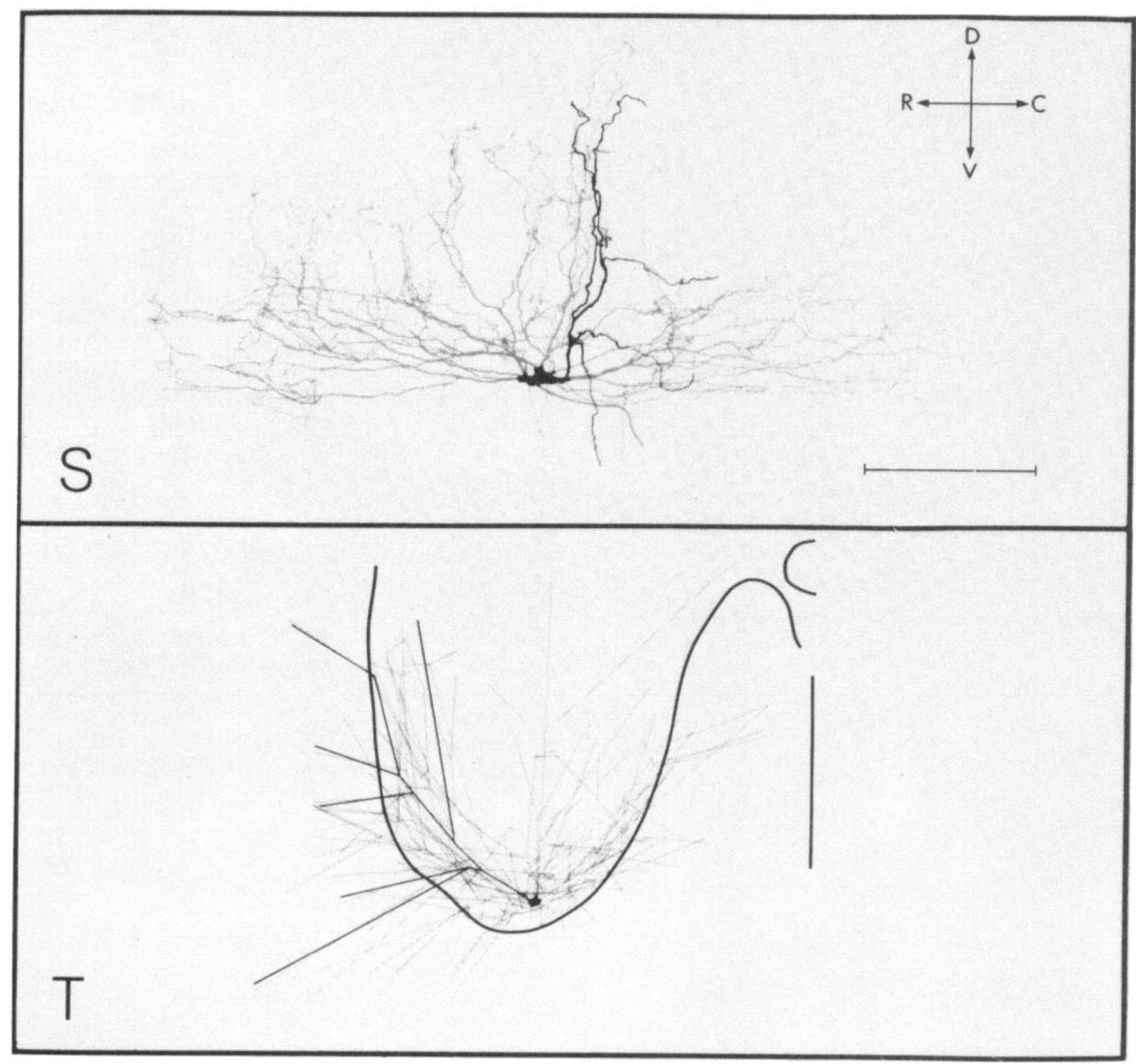

Figure 6. Distribution of a stem dendrite whose branches were restricted to the dorsolateral zone lateral to the soma.

a region which corresponds to the dorsomedial zone and the medial one-half of the dorsal zone of Figure 2 ( Nyberg-Hansen and Mascitti, 1964; Petras, 1967; Wilson and Yoshida, 1969a). Other descending systems, such as the interstitial spinal tract and medial vestibular spinal tract, also terminate in these zones, but their major regions of termination are usually more dorsal in lamina VII ( $\mathrm{Ny}$ berg-Hansen, 1964, 1966; Wilson and Yoshida, 1969b; Fukushima et al., 1978). Other descending systems, such as the medullary and pontine reticular formation, terminate over a wider area (Petras, 1967; Basbaum et al., 1978; Peterson et al., 1978, 1979; Martin et al., 1979). This area includes the dorsomedial, dorsal, and dorsolateral zones. Interestingly, no descending system which make monosynaptic connections on dorsal neck muscle motoneurons have been found to terminate in the region corresponding to the ventral zone (see, however, Holstege et al., 1979; Martin et al., 1981). The ventral zone does contain, however, the terminals of dorsal root afferents (Imai and Kusama, 1969) which do make monosyn- aptic connections on dorsal neck muscle motoneurons (Wilson and Maeda, 1974; Anderson, 1977; Rapoport, 1979). Thus, the territory occupied by the complete dendritic tree of biventer cervicis and complexus motoneurons may be divided into several zones not only according to the dendritic distribution pattern but also according to the arrangement of primary afferent and descending systems which make monosynaptic connections on these motoneurons.

One-half of the stem dendrites were distributed to only one zone. This finding suggests that these dendrites may receive a specific set of inputs and not just a smaller version of the input directed to the motoneuron as a whole. For example, stem dendrites whose branches were restricted to the ventral zone would be expected to receive connections only from primary afferents. These dendrites also could be influenced by descending systems but only via interneuronal pathways. This scheme of spatial segregation is similar to that proposed for many neurons, including motoneurons, whose dendrites appear 


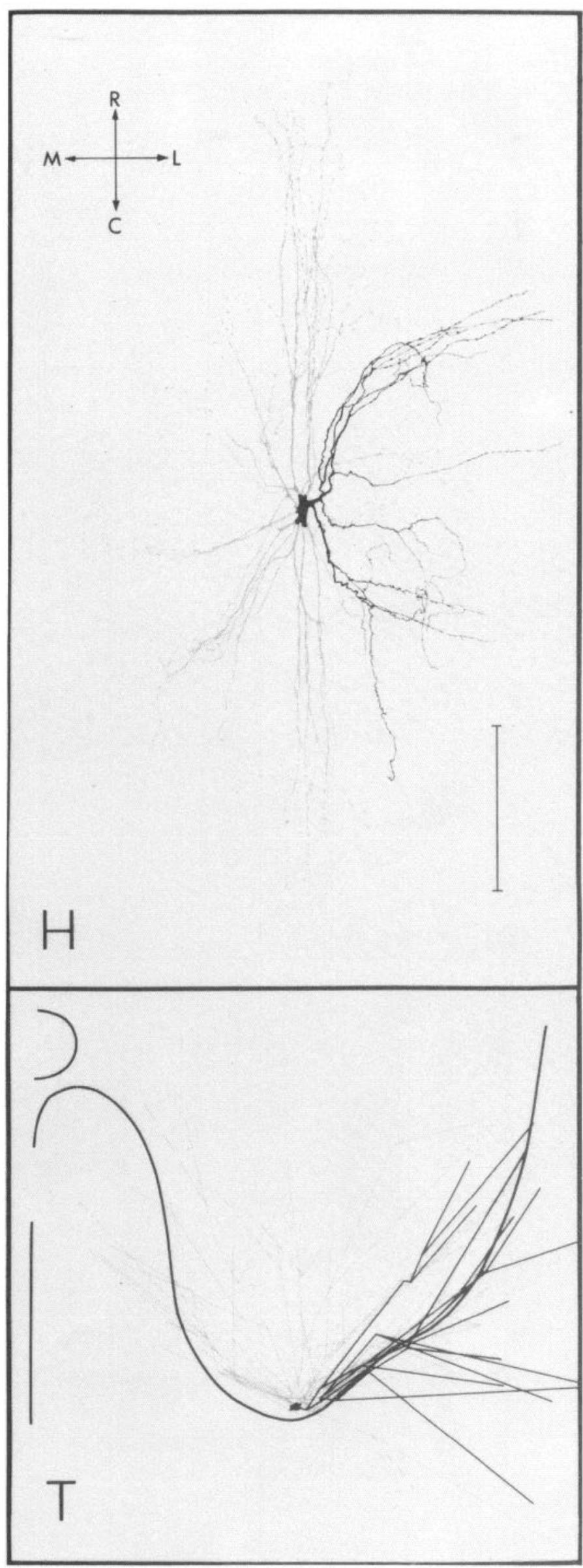

Figure 7. Distribution of a stem dendrite whose branches were restricted to the dorsolateral zone rostral and caudal to the soma.

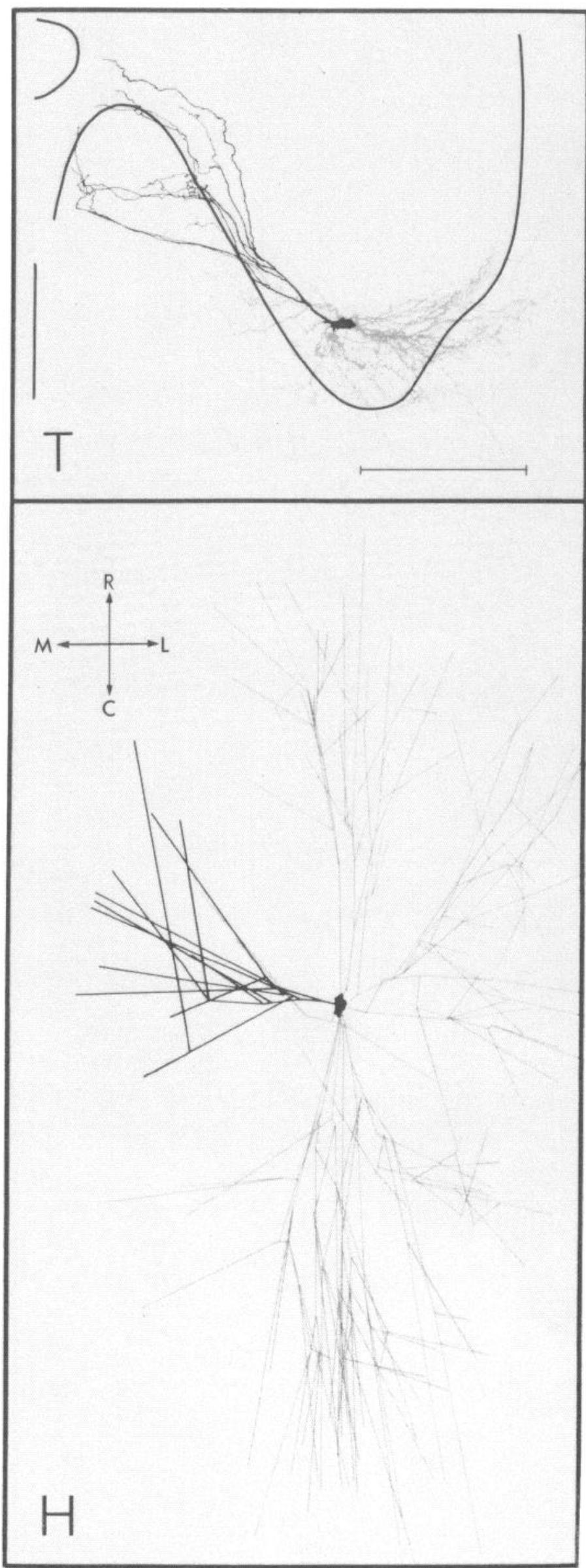

Figure 8. Distribution of a stem dendrite whose branches were restricted to the dorsomedial zone. 


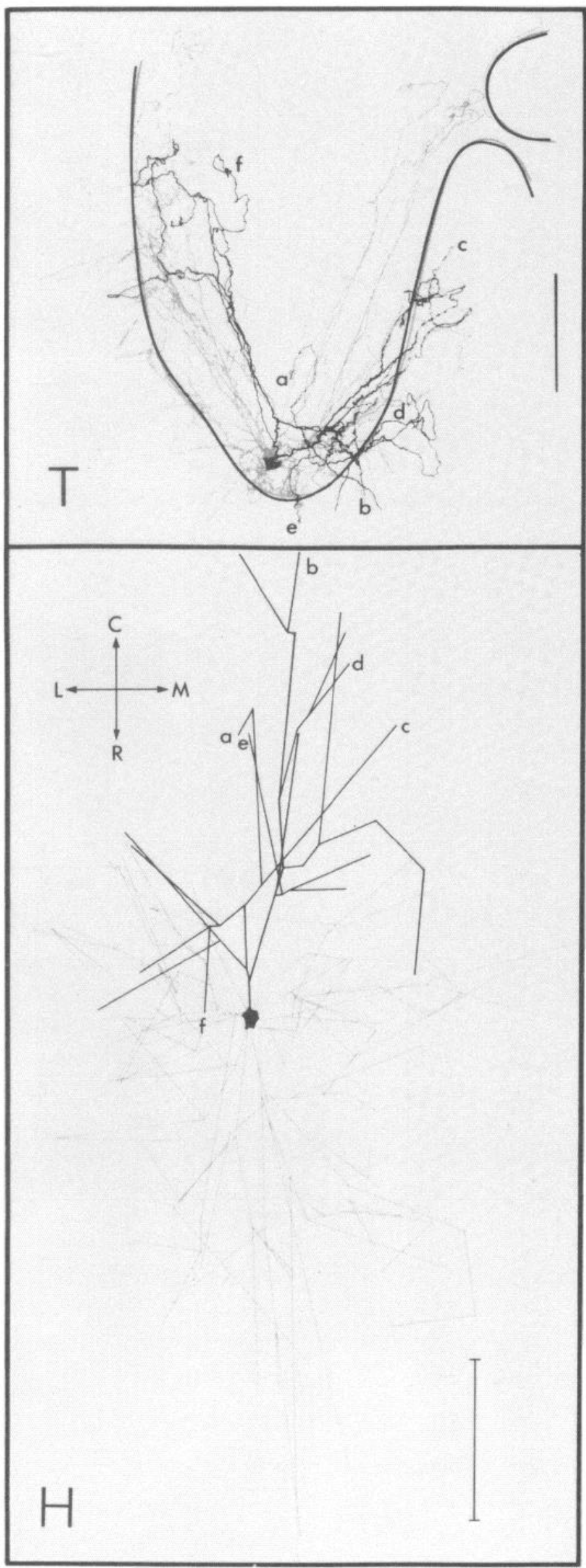

Figure 9. Distribution of a stem dendrite whose branches projected into three zones: the ventral, the dorsomedial, and dorsolateral zones.
TABLE I

Frequency of distribution patterns of biventer cervicis and complexus motoneuron stem dendrites

V, DL, DM, and D correspond to the regions shown in Figure 2.

\begin{tabular}{|c|c|c|c|c|c|c|}
\hline \multirow{2}{*}{$\begin{array}{c}\text { Distribution } \\
\text { Zone }\end{array}$} & \multicolumn{5}{|c|}{ Cell Number } & \multirow{2}{*}{$\begin{array}{l}\text { Total Number of } \\
\text { Stem Dendrites }\end{array}$} \\
\hline & 1 & 2 & 3 & 4 & 5 & \\
\hline V & 2 & 3 & 3 & 3 & 2 & 13 \\
\hline DL & 2 & 1 & 2 & 2 & 1 & 8 \\
\hline DM & 1 & 1 & 1 & 1 & & 4 \\
\hline D & & & & & & 0 \\
\hline Subtotal & 5 & 5 & 6 & 6 & 3 & 25 \\
\hline
\end{tabular}

$\begin{array}{llllll}\mathrm{V}+\mathrm{DL} & 1 & 1 & 1 & 1 & 4\end{array}$

$\begin{array}{llllll}\mathrm{V}+\mathrm{DM} & 2 & 1 & 2 & 2 & 7\end{array}$

$\mathrm{V}+\mathrm{D} \quad 1 \quad 1$

$\mathrm{DL}+\mathrm{DM}$

$\mathrm{DL}+\mathrm{D} \quad 1 \quad 1$

$\mathrm{DM}+\mathrm{D} \quad-\quad-\quad 2 \quad-\quad-\quad 2$

$\begin{array}{lllllll}\text { Subtotal } & 2 & 3 & 4 & 4 & 2 & \end{array}$

$\begin{array}{llllll}\mathrm{V}+\mathrm{DL}+\mathrm{DM} & 2 & 1 & 1 & 2 & 6\end{array}$

$\mathrm{V}+\mathrm{DL}+\mathrm{D} \quad 1 \quad 1$

$\mathrm{V}+\mathrm{DM}+\mathrm{D} 22$

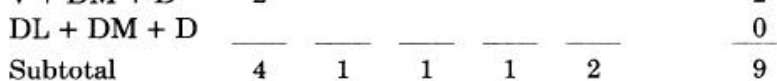

$\begin{array}{lllllll}\text { Total } & 11 & 9 & 11 & 11 & 7 & 49\end{array}$

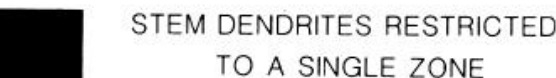

TO A SINGLE ZONE

MULTI-ZONE STEM

DENDRITES

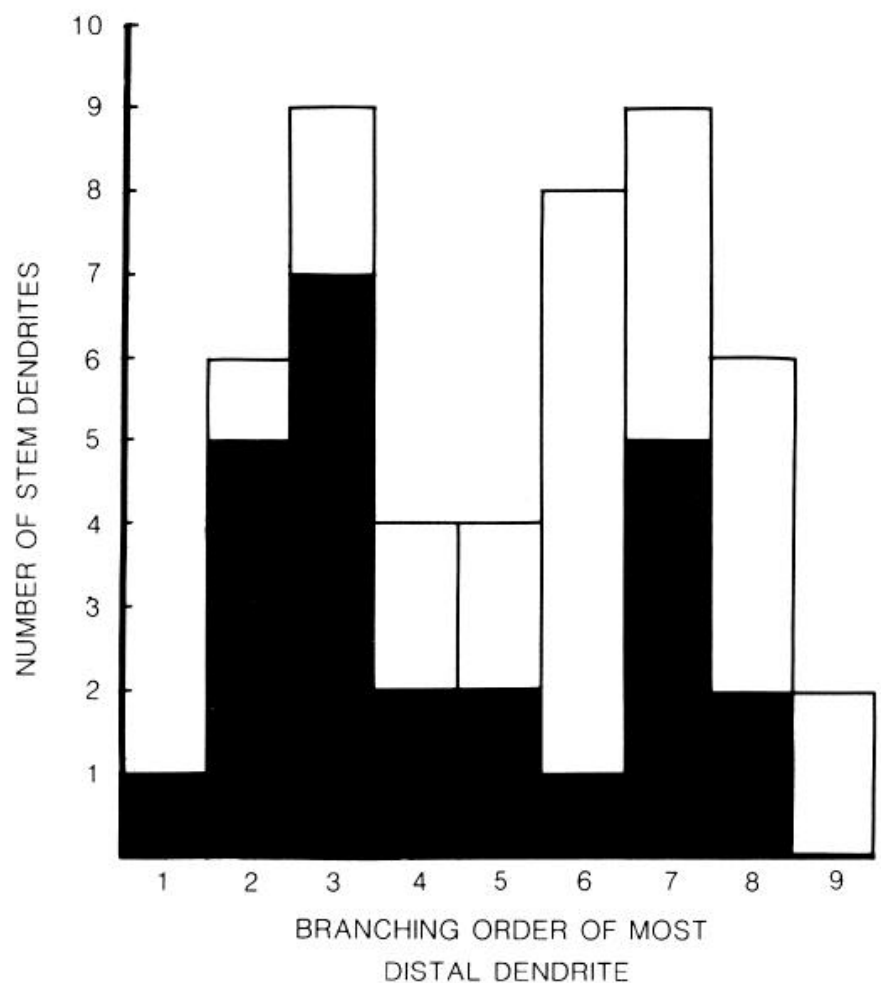

Figure 10. Maximum order of branching reached by the stem dendrites of biventer cervicis and complexus motoneurons ( $n$ $=49$ ). 


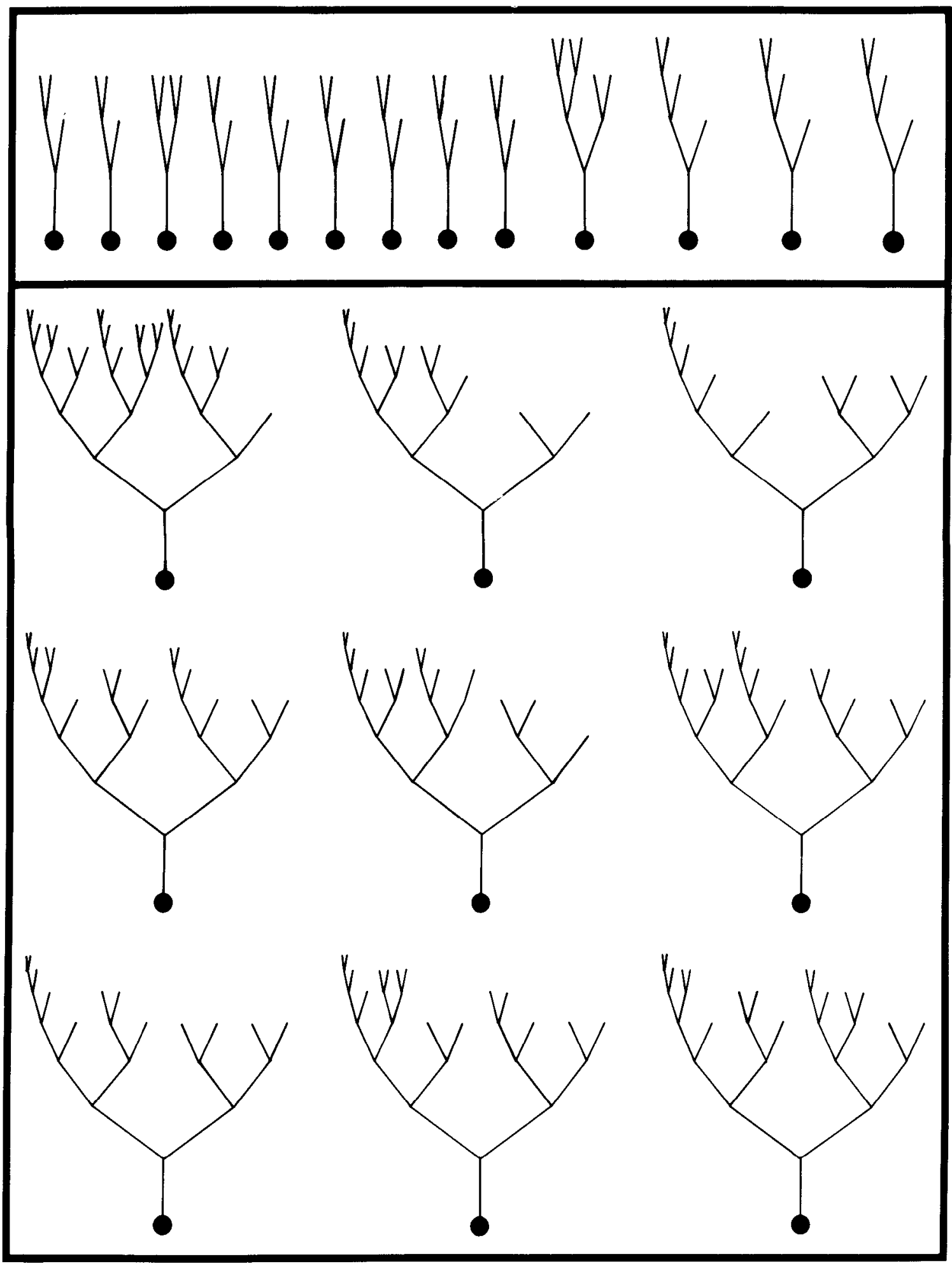

Figure 11. Examples of the branching structure of simple and complex stem dendrites. The upper drawing illustrates all stem dendrites whose maximum order of branching was three or four. The lower drawing illustrates all stem dendrites whose maximum order of branching was seven. Most of the simpler stem dendrites follow a similar pattern of dendritic branching. The more complex dendrites show a more variable pattern of dendritic branching. Note that the branching structure of the distal regions of the complex stem dendrites is similar to the branching structure of the entire branching pattern of the simple stem dendrites.

to be arranged in an organized fashion to receive specific inputs (Ramon y Cajal, 1909; Fadiga and Brookhart, 1960; Sprague and Ha, 1964; Scheibel and Scheibel, 1970b; Szekely, 1976; Shepherd, 1979; Bregman and Cruce, 1980; Egger et al., 1980). Nevertheless, three other characteristics of biventer cervicis and complexus motoneuron stem dendrites indicate that the integration of different inputs on the motoneuron surface cannot be a simple consequence of each stem dendrite receiving a different set of inputs. 


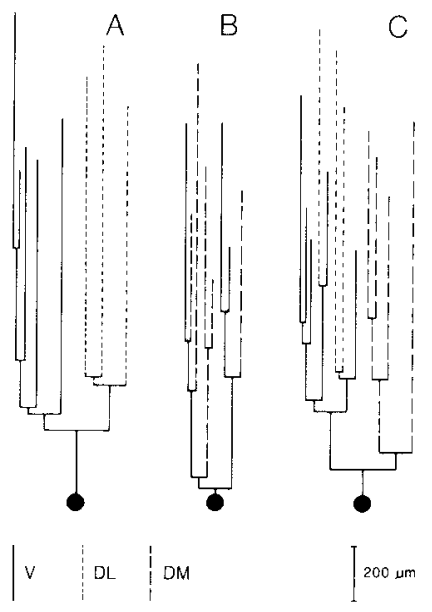

Figure 12. Schematic representation of three stem dendrites to show the relationship between the branching structure and distribution pattern. Only the vertical axis is drawn to scale. Dendritic lengths were measured using a cartographer's plan measure and include the distance traveled out of the plane of section. The solid lines indicate dendrites in the ventral $(V)$ zone, the short dashed lines indicate dendrites in the dorsolateral $(D L)$ zone, and the long dashed lines indicate dendrites in the dorsomedial $(D M)$ zone. A, Example of a stem dendrite with two major stalks. Branches from each stalk projected to separate zones. $B$, Example of a stem dendrite with two major stalks. Branches from both stalks projected into the same zones. $C$, Example of a stem dendrite with three major stalks. Branches from one stalk were restricted to the dorsomedial zone. Branches from the other two stalks intermingled in the ventral and dorsolateral zones.

(1) One-half of the stem dendrites examined in this study were distributed into two or more zones. Many of these dendrites projected to the ventral zone as well as a more dorsal zone, regions which receive different inputs. The integration of these inputs therefore will occur on the dendritic tree and not at the level of the soma as would occur for stem dendrites which project to a single zone. Moreover, the present study has shown that the distribution of dendritic branches to two or more zones can be the result of last order dendrites projecting to different zones. Thus, the site of integration of different inputs could be quite distal to the cell body. Finally, most multizone stem dendrites were distributed in three basic patterns. This suggests that these stem dendrites may organize the afferent systems terminating on dorsal neck muscle motoneurons into specific spatial patterns which could maximize (or minimize) interactions between certain sets of inputs.

(2) It was common, particularly for stem dendrites in the ventral zone, for 2 or more stem dendrites to share a common territory. It has been suggested that the arrangement of synaptic inputs on different dendritic branches can reduce significantly the nonlinear summation of postsynaptic potentials which occur when the same inputs are distributed on neighboring zones on the same dendritic branch (Rall, 1964; Rall et al., 1967; Rinzel and Rall, 1973). Thus, positioning 2 or more stem dendrites in the same zone may be an effective means of increasing the efficacy of synaptic transmission onto the motoneuron.

(3) Finally, some stem dendrites followed a path which traveled through two zones. For example, stem dendrites which projected to the ventral zone and either the dorsolateral or dorsomedial zones often followed an indirect pathway such that the dorsolateral or dorsomedial dendrites traveled for a considerable distance through the ventral zone before ascending and terminating in the dorsolateral or dorsomedial zone. Thus, the integration of different inputs on these dendrites could occur along the path followed by the dendrite.

The observations of the present study also raise questions regarding the universal application of the equivalent cylinder model to motoneuron dendritic trees. The distribution of branches of individual stem dendrites to different zones, which may, as a consequence, receive different inputs, is, in itself, not a major problem. Although inputs to the equivalent cylinder model are assumed to contact all dendrites equally, it is a relatively simple procedure to modify the model to accommodate different inputs to each stem dendrite individually (Rall and Rinzel, 1973; Rinzel and Rall, 1973; Redman, 1973). The present study also indicates that the branching structure of the stem dendrites of motoneurons is more complex than the symmetrical and equal branching structure used to develop the equivalent cylinder model. Once again, this observation may not pose a serious problem if the electrotonic lengths of daughter dendrites are the same (Rall, 1959). In order for this condition to be satisfied, the electrotonic lengths of the proximal side branches would have to equal the combined electronic length of the shaft and distal branches of the stalk from which the simple proximal side branch emerged. Thus, predictions of the cable properties of biventer cervicis and complexus motoneurons based on the equivalent cylinder model would be premature in the absence of more detailed information about the distribution of identified afferent systems and additional quantitative morphological studies.

\section{References}

Anderson, M. E. (1977) Segmental reflex inputs to motoneurons innervating dorsal neck musculature in the cat. Exp. Brain Res. 28: 175-187.

Barrett, J. N., and W. E. Crill (1974) Specific membrane properties of cat motoneurons. J. Physiol. (Lond.) 239: 301-324.

Basbaum, A. I., C. H. Clanton, and H. L. Fields (1978) Three bulbospinal pathways from the rostral medulla of the cat: An autoradiographic study of pain modulating systems. J. Comp. Neurol. 178: 209-224.

Bregman, B. S., and W. L. R. Cruce (1980) Normal dendritic morphology of frog spinal motoneurons: A Golgi study. J. Comp. Neurol. 193: 1035-1045.

Brown, A. G., and R. E. W. Fyffe (1981) Direct observations on the contacts made between Ia afferent fibres and $\alpha$-motoneurons in the cat's lumbrosacral spinal cord. J. Physiol. (Lond.) 313: 121-140.

Burke, R. E., B. Walmsley, and J. A. Hodgson (1979) HRP anatomy of group Ia afferent contacts on alpha motoneurons. Brain Res. 160: 347-352.

Egger, M. D., N. C. G. Freeman, and E. Proshansky (1980) Morphology of spinal motoneurones mediating a cutaneous spinal reflex in the cat. J. Physiol. (Lond.) 306: 349-363.

Fadiga, E., and J. M. Brookhart (1960) Monosynaptic activation of different portions of the motor neuron membrane. Am. J. Physiol. 198: 693-703.

Fukushima, K., N. G. Pitts, and B. W. Peterson (1978) Direct excitation of neck motoneurons by interstitiospinal fibers. Exp. Brain Res. 33: 565-581. 
Holstege, G., H. G. J. M. Kuypers, and R. C. Boer (1979) Anatomical evidence for direct brain stem projections to the somatic motoneural cell groups and automatic preganglionic cell groups in cat spinal cord. Brain Res. 171: 329-333.

Imai, Y., and T. Kusama (1969) Distribution of the dorsal root fibres in the cat. An experimental study with the Nauta method. Brain Res. 13: 338-359.

Jack, J. J. B., D. Noble, and R. W. Tsien (1975) Electric Current Flow in Excitable Cells, Clarendon Press, Oxford.

Keirstead, S., and P. K. Rose (1980) The distribution of dendrites from splenius motoneurons stained intracellularly with horseradish peroxidase. Can. J. Physiol. Pharmacol. 11: 89.

Light, A. R., and C. B. Metz (1978) The morphology of the spinal cord efferent and afferent neurons contributing to the ventral roots of the cat. J. Comp. Neurol. 179: 501-516.

Lux, H. D., and P. Schubert (1975) Some aspects of the electroanatomy of dendrites. Adv. Neurol. 12: 29-44.

Lux, H. D., P. Schubert, and G. W. Kreutzberg (1970) Direct matching of morphological and electrophysiological data in cat spinal motoneurons. In Excitatory Synaptic Mechanisms, P. Anderson and J. Jansen, eds., pp. 189-198, Universitets Forleget, Oslo, Norway.

Martin, G. F., A. O. Humbertson, Jr., L. C. Laxson, W. M Panneton, and I. Tschismadia (1979) Spinal projections from the mesencephalic and pontine reticular formation in the North American opossum: A study using axonal transport techniques. J. Comp. Neurol. 187: 373-400.

Martin, G. F., T. Cabana, A. O. Humbertson, Jr., L. C. Laxson, and W. M. Panneton (1981) Spinal projections from the medullary reticular formation of the North American opossum: Evidence of connectional heterogeneity. J. Comp. Neurol. 196: 663-682.

Nyberg-Hansen, R. (1964) Origin and termination of fibres from the vestibular nuclei descending in the medial longitudinal fasciculus. An experimental study with silver impregnation methods in the cat. J. Comp. Neurol. 122: 355-367.

Nyberg-Hansen, R. (1966) Sites of termination of interstitiospinal fibers in the cat. An experimental study with silver impregnation methods. Arch. Ital. Biol. 104: 98-111.

Nyberg-Hansen, R., and T. A. Mascitti (1964) Sites and mode of termination of fibers of the vestibulospinal tract in the cat. J. Comp. Neurol. 122: 369-387.

Peterson, B. W., N. G. Pitts, K. Fukushima, and R. Mackel (1978) Reticulospinal excitation and inhibition of neck motoneurons. Exp. Brain Res. 32: 471-489.

Peterson, B. W., N. G. Pitts, and K. Fukushima (1979) Reticulospinal connections with limb and axial motoneurons. Exp. Brain Res. 36: 1-20.

Petras, J. M. (1967) Cortical, tectal and tegmental fiber connections in the spinal cord of the cat. Brain Res. 6: 275-324.

Rall, W. (1959) Branching dendritic trees and motoneuron membrane resistivity. Exp. Neurol. 1: 491-527.

Rall, W. (1964) Theoretical significance of dendritic trees for neuronal input-output relations. In Neural Theory and Modelling, R. Reiss, ed., pp. 73-97, Stanford University Press, Stanford, CA.

Rall, W. (1977) Core conduction theory and cable properties of neurons. In Handbook of Physiology. Sect. 1: The Nervous System, E. R. Kandel, ed., Vol. 1, pp. 39-98, American Physiological Society, Bethesda, MD.

Rall, W., and J. Rinzel (1973) Branch input resistance and steady attenuation for input to one branch of a dendritic: neuron model. Biophys. J. 13: 648-688.

Rall, W., R. E. Burke, T. G. Smith, P. G. Nelson, and K. Frank (1967) Dendritic location of synapses and possible mechanisms for the monosynaptic EPSP in motoneurons. J. Neurophysiol. 30: 1169-1193.

Ramon y Cajal, S. (1909) Histologie du Systeme Nerveux de
l'Homme et des Vertebres, Vol. 1, Maloine, Paris.

Rapoport, S. R. (1979) Reflex connexions of motoneurones of muscles involved in head movement in the cat. J. Physiol. (Lond.) 289: 311-327.

Redman, S. J. (1973) The attenuation of passively propagating dendritic potentials in a motoneurone cable model. J. Physiol. (Lond.) 234: 637-664.

Rinzel, J., and W. Rall (1973) Transient response in a dendritic neuron model for current injected at one branch. Biophys. J. 14: 759-790.

Romanes, G. J. (1964) The motor pools of the spinal cord. Prog. Brain Res. 11: 93-119.

Rose, P. K. (1981) Distribution of dendrites from biventer cervicis and complexus motoneurons stained intracellularly with horseradish peroxidase in the adult cat. J. Comp. Neurol. 197: 395-409.

Rose, P. K., and F. J. R. Richmond (1981) White matter dendrites in the upper cervical spinal cord of the adult cat: A light and electron microscopic study. J. Comp. Neurol. 199: 191-203.

Rose, P. K., and D. A. S. Twiddy (1981) The organization of stem dendrites of dorsal neck muscle motoneurons in the adult cat. Soc. Neurosci. Abstr. 7: 898.

Rose, P. K., S. V. Vanner, and R. Hindle (1981) Variations in dendritic distribution patterns of motoneurons innervating the same muscle: An intracellular HRP study of trapezius motoneurons in the adult cat. Physiol. Can. 12: 130.

Scheibel, M. E., and A. B. Scheibel (1966) Spinal motoneurons, interneurons and Renshaw cells. A Golgi study. Arch. Ital. Biol. 104: 328-353.

Scheibel, M. E., and A. B. Scheibel (1967) A structural analysis of spinal interneurons and Renshaw cells. In The Interneuron, M. Brazier, ed., pp. 159-208, University of California Press, Berkeley, CA.

Scheibel, M. E., and A. B. Scheibel (1969) Terminal patterns in cat spinal cord. III. Primary afferent collaterals. Brain Res. 13: $417-443$.

Scheibel, M. E., and A. B. Scheibel (1970a) Organization of spinal motoneuron dendrites in bundles. Exp. Neurol. 28: 106-112.

Scheibel, M. E., and A. B. Scheibel (1970b) Of pattern and place in dendrites. Int. Rev. Neurobiol. 1: 1-26.

Shepherd, G. M. (1979) The Synaptic Organization of the Brain, 2nd ed., Oxford University Press, Oxford.

Sprague, J. M., and H. Ha (1964) The terminal fields of dorsal root fibres in the lumbrosacral spinal cord of the cat, and the dendritic organization of the motor nuclei. Prog. Brain Res. 11: $120-154$.

Szekely, G. (1976) The morphology of motoneurons and dorsal root fibers in the frog's spinal cord. Brain Res. 103: 275-290.

Ulfhake, B., and S. Cullheim (1981) A quantitative light microscopic study of the dendrites of cat spinal $\gamma$-motoneurons after intracellular staining with horseradish peroxidase. J. Comp. Neurol. 202: 585-596.

Ulfhake, B., and J. -O. Kellerth (1981) A quantitative light microscopic study of the dendrites of cat spinal $\alpha$-motoneurons after intracellular staining with horseradish peroxidase. J. Comp. Neurol. 202: 571-583.

Wilson, V. J., and M. Maeda (1974) Connections between semicircular canals and neck motoneurons in the cat. J. Neurophysiol. 37: 346-357.

Wilson, V. J., and M. Yoshida (1969a) Comparison of effects of stimulation of Deiter's nucleus and medial longitudinal fasciculus on neck, forelimb and hindlimb motoneurons. J. Neurophysiol. 32: 743-759.

Wilson, V. J., and M. Yoshida (1969b) Monosynaptic inhibition of neck motoneurons by the medial vestibular nucleus. Exp. Brain Res. 9: 365-380. 